\title{
EL INSTITUTO NACIONAL DE ENFERMEDADES NEOPLÁSICAS EN EL CONTROL DEL CÁNCER EN EL PERÚ
}

\author{
THE ROLE OF THE NATIONAL INSTITUTE OF NEOPLASTIC DISEASES IN THE CONTROL \\ OF CANCER IN PERU
}

\author{
Miriam Rosario Salazar, ,a, Roxana Regalado-Rafael 1,b, Jeannie Magalli Navarro ,c, Dayana Melissa Montanez ${ }^{1, d}$, \\ Julio Elías Abugattas ${ }^{1, e}$, Tatiana Vidaurre ${ }^{1, f}$ \\ Instituto Nacional de Enfermedades Neoplásicas. Lima, Perú. \\ a Obstetriz; ${ }^{b}$ médico patologo clínico; ${ }^{c}$ nutricionista; ${ }^{\mathrm{d}}$ bióloga; ${ }^{\mathrm{e}}$ médico cirujano oncólogo y cirujano de mamas y piel magister en Medicina; ${ }^{\mathrm{f}}$ médico \\ oncólogo especialista en gestión de servicios en salud magíster en Oncología Molecular \\ Recibido: 04-02-13; Aprobado: 06-03-13
}

\begin{abstract}
RESUMEN
La incidencia estimada de cáncer en el Perú es de 150 casos x 100000 habitantes. Este ocupa el segundo lugar de las causas mortalidad a nivel nacional y se estima que el $75 \%$ de los casos se diagnostican en etapa avanzada y principalmente en Lima. En ese contexto, el Instituto Nacional de Enfermedades Neoplásicas (INEN) ha promovido la descentralización de la atención oncológica con la creación de los institutos regionales de enfermedades neoplásicas, las unidades oncológicas y los preventorios. Asimismo ha diseñado, desarrollado e implementado el Programa Presupuestal de Prevención y Control de Cáncer, por lo que desde el año 2011, más de 7000 establecimientos del país asignan recursos para la prevención, promoción y detección precoz de los cánceres más frecuentes en el Perú. Con el financiamiento del seguro estatal, se integraron los ejes estratégicos básicos para una atención integral del cáncer en la población de menores recursos. De esta manera y dentro de una política de estado integradora y articulada con el sector salud, nace el Plan Nacional para la Atención Integral del Cáncer y el Mejoramiento del Acceso a los Servicios Oncológicos en el Perú, denominado "Plan Esperanza". En este artículo, desarrollamos el papel que viene cumpliendo el INEN en el control del cáncer como problema de salud pública, destacando la importancia del Programa Estratégico Presupuestal de Prevención y Control del Cáncer y de su papel en el "Plan Esperanza".
\end{abstract}

Palabras clave: Cáncer; Salud pública; Prevención; Promoción de la salud; Descentralización; Atención integral de salud; Programas nacionales de salud; Cobertura universal (fuente: DeCS BIREME).

\begin{abstract}
With a mortality rate that constitutes the second nationwide, the estimated incidence of cancer in Peru is 150 cases $\times 100000$ inhabitants. Around $75 \%$ of the cases are diagnosed at an advanced stage and mainly in Lima. In this context, the National Institute of Neoplastic Diseases (INEN) has promoted the decentralization of oncological care creating regional institutes of neoplastic diseases, oncological units and prevention centers. In addition, INEN has designed, developed and implemented the Budgetary Program for Cancer Prevention and Control, which, since 2011, has allowed for more than 7000 centers around the country to allocate resources to the prevention, promotion and early detection of the most frequent cancers in Peru. With the financial support of the state's health insurance system, the basic strategic central points were integrated to provide low-income cancer patients with comprehensive medical care. Through this way, and within the framework of a state policy integrated to and articulated with the health sector, the National Plan for Comprehensive Medical Care for Cancer Patients and the Improvement in the Access to Oncological Services in Peru, known as "The Hope Plan", was born. This article elaborates on the role that INEN plays in the control of cancer as a public health issue, highlighting the importance of the Strategic Budgetary Program for Cancer Prevention and Control and its role in the "The Hope Plan".
\end{abstract}

Key words: Cancer; Public health; Prevention; Health promotion; Decentralization; Comprehensive health care; National health programs; Universal coverage (source: MeSH NLM). 


\section{INTRODUCCIÓN}

De acuerdo con los datos del Instituto Nacional de Enfermedades Neoplásicas, se estima que la incidencia anual del cáncer en el Perú es de 150 casos cada 100 000 habitantes aproximadamente, lo que correspondería a 45000 nuevos casos por cada año. Actualmente, el mayor porcentaje de los diagnósticos de cáncer se realiza cuando la enfermedad se encuentra en etapa avanzada, alcanzando el $75 \%$ de los casos diagnosticados. Este hecho conlleva a una menor probabilidad de curación, menor calidad de vida, un costo de tratamiento mayor y una elevada mortalidad. La pobreza es un factor que se relaciona con esta detección tardía del cáncer, debido a las dificultades de acceso a los servicios de salud, a una escasa cultura de prevención, a la fuerte influencia de estilos de vida poco saludables, al bajo nivel de educación, entre otros ${ }^{(1)}$.

El Registro de Cáncer de Lima Metropolitana, muestra que los tipos de cáncer más frecuentes son: estómago, mama, cérvix, próstata y pulmón. Según la estadística del Instituto Nacional de Estadística e Informática la población del Perú asciende a 28220764 habitantes, según los resultados del XI Censo de Población (2) y según las estadísticas del MINSA ${ }^{(3)}$ el cáncer ocupa el segundo lugar de mortalidad nacional con un importante impacto socioeconómico.

La promoción de la salud, la prevención, el diagnóstico precoz, y el tratamiento integral y oportuno son las estrategias fundamentales para el control del cáncer a nivel nacional. La promoción a través de la educación para la salud y el fomento de estilos de vida saludables inciden sobre los factores determinantes de la salud anticipándose a la aparición de riesgos en la población a fin de evitar el incremento de las enfermedades neoplásicas.

Para hablar de tratamiento de cáncer en el Perú, se debe considerar el factor de desigualdad social existente, en particular entre las personas pobres y extremo pobres que habitan zonas rurales. En este contexto, afrontar un tratamiento óptimo para el cáncer resulta muy difícil por representar un enorme gasto económico para el paciente y su entorno familiar ${ }^{(4)}$. Es por ello que es necesario adoptar medidas que eviten el incremento de los casos de cáncer, no solo por la prevención a la exposición de los factores de riesgo sino considerando la adquisición de hábitos saludables en las diferentes etapas de la vida como parte de la promoción de salud. Asimismo, es importante trabajar en medidas que permitan disminuir la carga de sufrimiento de las personas con cáncer y brindar una adecuada calidad de vida. Ello implica brindar tratamientos óptimos, un soporte integral que acompañe al paciente durante el proceso de su enfermedad y los cuidados paliativos en pacientes terminales; considerando la atención oncológica desde un enfoque integral ${ }^{(5)}$.

El 13 de mayo de 1939 se funda el Instituto Nacional del Cáncer, posteriormente denominado Instituto Nacional de Enfermedades Neoplásicas (INEN), que en sus 73 años de vida institucional, se ha consolidado como una institución científica de alta especialización, cuenta con una amplia trayectoria nacional e internacional y realiza un intenso trabajo en el desarrollo de conocimiento técnico especializado en cáncer. Atiende un promedio de 16 mil pacientes nuevos en 86 consultorios externos y 378 camas hospitalarias, con un permanente crecimiento de su capacidad resolutiva para afrontar los problemas de la especialidad. Debido al incremento de la demanda de atención oncológica en el país fue necesario plantear una nueva forma de lidiar con el cáncer, es así que desde el año 2003 se consideró indispensable trabajar acciones sanitarias efectivas para el control de esta enfermedad, dentro del marco del rol del estado en la salud pública nacional, por eso desde el año 2004 , mediante Ley 28343 se declara de interés y necesidad publica la descentralización de los servicios oncológicos, disponiendo la creación de servicios o sedes macrorregionales ${ }^{(6)}$.

\section{CONSOLIDACIÓN DEL INEN EN LA GESTIÓN DE LAS POLIITICAS DE PREVENCIÓN Y CONTROL DEL CÁNCER EN EL PERÚ}

En el año 2006, mediante Ley 28748 , se creó como organismo público descentralizado el INEN y mediante el Decreto Supremo 001-2007. S.A. se aprobó el reglamento de organización y funciones en el cual se establecen como funciones generales del INEN (7):

- Establecer las políticas y objetivos estratégicos nacionales y los objetivos, estrategias, programas y proyectos institucionales en materia oncológica, para la promoción, prevención, protección, diagnóstico y tratamiento del cáncer en los diferentes niveles de complejidad de atención, rehabilitación de capacidades afectadas por el cáncer y/o su tratamiento, mejora de calidad de vida, y otros procesos asistenciales interrelacionados.

- Ejercer la rectoría técnica y emitir opinión técnica en materia oncológica en el ámbito nacional.

En dicho documento, además, se señala que el INEN tiene la misión de proteger, promover, prevenir y garantizar la atención integral del paciente oncológico, dando prioridad a las personas de escasos recursos económicos; así como controlar, técnica y 
administrativamente a nivel nacional, los servicios de salud de las enfermedades neoplásicas, y realizar las actividades de investigación y docencia ${ }^{(7)}$.

Igualmente, en el año 2006, mediante la Resolución Ministerial 003-2007/MINSA, se aprobó el plan nacional para el fortalecimiento para la prevención y control del cáncer en el país ${ }^{\left({ }^{(8)}\right.}$ y en el año 2007 se aprobó el plan concertado en salud (9) ${ }^{(9)}$ yo objetivo general es la disminución de la mortalidad por cáncer, teniendo como prioridad el cáncer de cérvix, mama, estómago y próstata. En este marco, también en ese año, se aprobó el plan esencial de aseguramiento en salud (PEAS) mediante el D.S. 016-2009-S.A. ${ }^{(10)}$, como plan mínimo a nivel nacional, que contiene las condiciones y prestaciones en el cual se toman en cuenta los siguientes tipos de cáncer: cérvix (incluye la displasia cervical), mama, colon, estómago y próstata.

En este escenario existieron algunas iniciativas colaborativas de la American Cancer Society, la Unión Internacional Contra el Cáncer y el Instituto Nacional del Cáncer de los Estados Unidos las cuales junto al INEN, el Ministerio de Salud (MINSA), el seguro social (EsSalud), las fuerzas armadas y policiales, las organizaciones no gubernamentales (como la Liga de Lucha Contra el Cáncer), la Fundación Peruana del Cáncer, los voluntariados, la Sociedad Peruana de Cancerología, la Municipalidad de San Borja (en representación de la Red de Municipios Saludables), la Organización Panamericana de Salud, entre otros, constituyeron la Coalición Multisectorial Perú Contra el Cáncer con la visión de un Perú libre de cáncer avanzado con cultura y educación en salud y con acceso a servicios de calidad para el control del cáncer al 2016, pero que no logró acceder a un presupuesto para este fin.

En el año 2011, a lo dispuesto en la Ley 29626 "Ley de Presupuesto del Sector Público para el Año Fiscal 2011" (11) se le asigna la suma de 28 millones de nuevos soles al INEN para implementar el programa estratégico de prevención y control de cáncer en diez regiones del país. En él se plantea como resultado final disminuir la tasa de morbimortalidad por cáncer en la población mayor de 18 años, priorizando la atención preventiva en los siguientes tipos de cáncer: cérvix, mama, pulmón, estómago y próstata. Es importante mencionar que el presupuesto asignado para ese primer año de implementación ha sido priorizado a las diez regiones con criterio de oportunidad demográfica, epidemiológica y de recursos disponibles, a saber: Lambayeque, La Libertad, Piura, Huánuco, Ica, Junín, Arequipa, Cusco, Callao y Lima, con diez hospitales centrales que van desde los niveles II-1 a III-1; dos institutos regionales de salud que son nivel III-2, y 64 establecimientos de salud del nivel 1-4, situación que en el proceso de la implementación en cada región, y de acuerdo a su realidad, se está ampliando hasta nivel 1-2, de acuerdo con las necesidades de cada región y al presupuesto asignado.

Siguiendo con la normativa nacional, con fecha 15 de marzo de 2011, mediante RM 178-2011 MINSA, se aprobó el documento técnico "Definiciones operacionales y criterios de programación de prevención y control del cáncer", tras el cual la Ley 29812 "Ley de Presupuesto del Sector Público para el año 2012" consigna al INEN un monto de cerca de 75 millones de nuevos soles con el objeto de transferirlo a los gobiernos regionales, para efecto del "Programa presupuestal prevención y control del cáncer".

Es importante mencionar también que en los últimos años se ha notado que la mayoría de los pacientes del INEN tienen condición de pobreza, por este motivo el Seguro Integral de Salud (SIS) y el INEN han venido trabajado en forma colaborativa buscando soluciones efectivas; a modo de ejemplo, durante los primeros 6 meses del año 2012, alrededor de 6100 pacientes con cáncer han sido tratados en los diversos servicios del INEN de manera gratuita, bajo la modalidad de financiamiento de casos especiales o cobertura extraordinaria del SIS.

\section{EL INEN EN LA DESCENTRALIZACIÓN DE LA ATENCIÓN DEL CÁNCER}

En este marco, el INEN ha promovido y acompañado los procesos de descentralización de la atención oncológica especializada a través de la creación del Instituto Regional de Enfermedades Neoplásicas Norte (IREN Norte), en Trujillo; del Instituto Regional de Enfermedades Neoplásicas Sur (IREN Sur), en Arequipa; de las unidades oncológicas en diversos establecimientos de salud del país, y de los preventorios (principalmente municipales) ubicados a nivel nacional. Asimismo, recientemente ha acompañando al gobierno regional de Junín en la elaboración y aprobación de su perfil de inversión y estudio de factibilidad del IREN Centro que ya se encuentra viable; en esta misma lógica se ha planteado la posibilidad de un IREN Amazónico, en Loreto.

De igual manera, con este programa presupuestal de prevención y control de cáncer, en el año 2011 y en el 2012, más de 7000 establecimientos del país tienen la oportunidad de presupuestar y asignar recursos para la prevención de cáncer y promoción de la salud, así como para la detección precoz de este problema de salud, 
en los cinco cáncer más frecuentes: estomago, mama, cérvix, próstata y pulmón; teniendo una ejecución sobresaliente.

El programa presupuestal ha beneficiado a 2650000 pobladores a nivel nacional, así mismo, se ha capacitado en prevención primaria de cáncer a 2794 profesionales de la salud a nivel nacional, y en prevención secundaria a 250 profesionales de la salud, principalmente médicos y obstetras de diferentes regiones del país.

El éxito de este programa sirvió como base para la ampliación de productos que permitan una atención integral del cáncer en el año 2013, es así que en el mes de junio de 2012 se presenta al Ministerio de Economía y Finanzas una ampliación de las actividades dentro del programa de prevención y control del cáncer ampliando el tratamiento a diez tipos de cáncer, se añaden a los cinco mencionados los de hígado, piel (no melanoma), colon y recto, linfoma y leucemia. Con lo que los diferentes establecimientos de país tienen la posibilidad de presupuestar recursos para promoción, prevención, detección temprana y tratamiento del cáncer, según sus competencias y capacidades resolutivas.

\section{MODELO SOCIAL MULTICAUSAL DEL CANCER}

Por otro lado, es importante mencionar que el INEN es la institución referente especializada en cáncer del Perú, en él convergen la multiculturalidad y la multietnicidad característica de nuestro país pues el $51 \%$ los pacientes provienen de provincia, estas características aunadas a las motivaciones por las que buscan atención podrían influir en la calidad de la atención y el buen trato, ya que las brechas culturales pueden acentuarse y ello generaría exclusión. La implementación de acciones de salud culturalmente sensibles implica una aproximación a las miradas más subjetivas que tiene la población, identificar sus recursos, revalorar el rol activo de las personas usuarias de los servicios, de tal manera que se puedan establecer alianzas que contribuyan a favorecer el bienestar y satisfacer las expectativas de la población $(12,13)$. En ese sentido, se está cambiando paradigmas mirando al cáncer como un concepto global de salud.

Desde el enfoque social multicausal (Figura 1), el cáncer no solo representa un problema de salud pública tradicional sino es un problema sociocultural de signos y significados construidos en la sociedad, por lo que necesita un cambio de paradigma que lo desmitifique, siendo ello fundamental para mejorar la oportunidad del acceso a los servicios oncológicos con una atención oportuna y de calidad, integrando los conceptos de equidad y respeto a la dignidad del ser humano; al atenderse en su propia localidad y su propia cultura, o cambiando el modelo de decisiones informadas a decisiones compartidas; además de establecerse un sistema de protección efectivo para el paciente, con un modelo de redes de referencia y contrarreferencia que le permita avanzar acompañado por el sistema, reorientando así la atención de salud de un manera dinámica y cálida, claro está que este es un proceso con un camino largo por recorrer ${ }^{(14,15)}$.

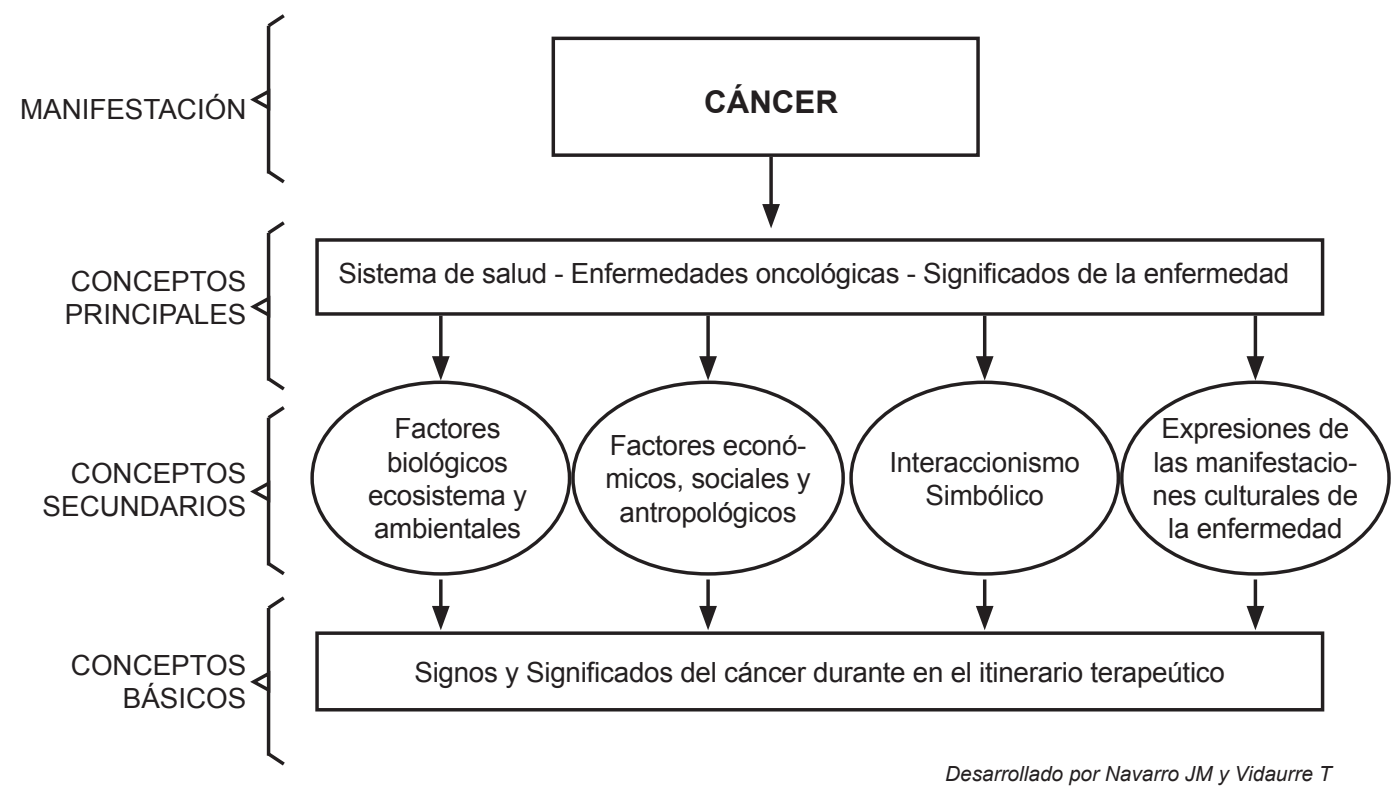

Figura 1. Modelo social multicausal del cáncer. 


\section{EL "PLAN ESPERANZA", LA NUEVA Y MEJOR OPORTUNIDAD}

Con este marco lógico se presentó una magnífica oportunidad para integrar los ejes estratégicos básicos que permitieran una atención integral del cáncer en la población de menores recursos: el programa presupuestal de prevención y control del cáncer del INEN y el SIS, con un gran plan nacional que genere una política de estado integradora y articuladora dentro del sector salud.

Es así que nace el Plan Nacional para la Atención Integral del Cáncer y el Mejoramiento del Acceso a los Servicios Oncológicos en el Perú, denominado "Plan Esperanza". Aprobado con el Decreto Supremo 009-2012 ${ }^{(16)}$, declara de interés nacional la atención integral del cáncer y el mejoramiento del acceso a los servicios oncológicos en el Perú.

Las estrategias de intervención sanitaria del Plan Esperanza, dentro de un análisis académico, podrían explicarse en cinco componentes, todos ellos tienen como propósito prevenir la aparición del cáncer en la población pobre y pobre extrema del Perú, a través de la promoción, prevención y detección precoz de casos con la finalidad de disminuir la morbilidad y mortalidad por cáncer en la población general y fortalecer la capacidad de oferta de servicios oncológicos del sector público. A continuación se presentan cada uno estos componentes.

\section{COMPONENTE PROMOCIONAL Y DE ACCIÓN INTERSECTORIAL}

El cual busca desarrollar y promover hábitos saludables en los individuos, familias, escuelas y centros de trabajo. Como parte de él se desarrollarán campañas de difusión y promoción, con la intención de generar impacto en la opinión pública, fortalecer el rol rector y la normatividad multisectorial, lograr la extensión de medidas sanitarias para la prevención y detección del cáncer, fortalecer el control de noxas ambientales, fortalecer la normatividad en relación al control de alimentos, y promocionar las condiciones laborales saludables. Para ello, se deberá fomentar el esfuerzo conjunto de los diferentes niveles de gobierno.

\section{COMPONENTE DE ATENCIÓN INTEGRAL PREVENTIVA Y ASISTENCIAL}

Dentro del cual se procurará desarrollar actividades ligadas a la detección precoz de cáncer en la población del primer nivel de atención (mediante un sistema de atención primaria especializada), mejorar las competencias clínicas del personal, desarrollar alianzas con otros prestadores para complementar la oferta especializada existente (especialmente con EsSalud). Además se buscará generar la atención de los casos detectados en todos los estadios. Para ello, se deberá organizar los servicios de salud por niveles de atención, fortalecer la oferta de servicios oncológicos tales como cirugía, quimioterapia, radioterapia, apoyo al diagnóstico y servicios afines. Dentro de este componente, además, se desarrollarán sistemas de acompañamiento para referencia y contrarreferencia según el grado de complejidad.

\section{COMPONENTE TÉCNICO NORMATIVO Y ORGANIZATIVO}

Será el responsable de vincular, como estrategia de intervención, la organización de los servicios de salud por niveles de atención y según ámbito de competencias. Las estrategias de intervención básicas para este componente técnico - normativo y organizativo involucran el desarrollo de la cartera de servicios oncológicos en el primer nivel de atención, el desarrollo de guías de práctica clínica para los principales cánceres, en todos los niveles de atención y el desarrollo de los flujos de atención para la referencia y contrarreferencia de los pacientes oncológicos.

\section{COMPONENTE DE GESTIÓN DE RECURSOS PRESUPUESTALES}

El personal a cargo de este componente realizará la estimación de recursos asignados para la atención de cáncer en las regiones y buscará generar un fondo de tratamiento de enfermedades oncológicas de alto costo en el SIS, denominado Fondo Intangible Solidario de Salud (FISSAL), con el cual se podrá contar con instrumentos de transferencia (cápita, tarifas, mecanismos de pago según nivel de resolución, diagnósticos resueltos, entre otros). Así mismo, deberá velar por los mecanismos de la auditoría clínica y de servicios, además de la transferencia condicionada de los recursos en función de resultados de producción y de gestión (registro de información, referencia oportuna, pacientes debidamente diagnosticados, pacientes tratados oportunamente, personas controladas previamente, entre otros).

\section{COMPONENTE DE DESCENTRALIZACIÓN Y DESCONCENTRACIÓN DE LOS SISTEMAS DE ALTA COMPLEJIDAD}

Que deberá impulsar la lógica de los institutos regionales especializados y el fortaleciendo del equipamiento con tecnología de avanzada para el acceso a tratamientos altamente especializados en el INEN. Asimismo, 
promoverá la educación e investigación para mantener un alto nivel de competencias profesionales especializadas mediante un intercambio científico permanente que permita las buenas prácticas en la atención oncológica.

El año 2012, el INEN inició una nueva etapa en la lucha contra el cáncer en el Perú, que se enmarca en el Plan Esperanza, pero que a la vez se enriquece con toda la experiencia obtenida en su larga trayectoria institucional. Para dar vida al Plan Esperanza el INEN ha sido el primer establecimiento que ha firmado convenio con el FISSAL, con lo cual se podrá garantizar el financiamiento del tratamiento del cáncer de los pacientes de menores recursos que se encuentran afiliados al SIS. Gracias a este convenio, el FISSAL otorga al INEN los recursos por adelantado con el fin de garantizar el oportuno abastecimiento, con lo cual se garantiza que a los pacientes oncológicos no les faltará ni los medicamentos ni los insumos para su tratamiento, el proceso de rehabilitación también se encuentra garantizado. La cobertura del Plan Esperanza alcanza siete tipos de cáncer (estómago, mama, cérvix, próstata, colon y recto, linfoma y leucemia) e incluye tratamientos altamente especializados como el trasplante de médula ósea. Se estima que para el 2013 el número de beneficiados, solo en el INEN, alcanzará los 19500 pacientes.

Es muy importante señalar que uno de los pilares fundamentales en los cuales se sostiene el Plan Esperanza, es el Programa Estratégico Presupuestal de Prevención y Control del Cáncer que ha sido diseñado, desarrollado e implementado por el INEN. La exitosa ejecución de este programa parece estar ligada al hecho de que la conducción sea por el ente técnico especializado, permitiendo mejores oportunidades para un trabajo operativo más dinámico y efectivo, con un enfoque basado en experiencias especializadas y conocimientos profundos sobre el cáncer, que realmente significa un cambio de paradigma en el modelo de salud pública del país ${ }^{(17)}$.

\section{NUEVOS SERVICIOS ONCOLOGICOS EN EL INEN}

Una necesidad de gran importancia en la salud pública nacional ha sido la demanda de un servicio de trasplante de médula ósea. EI INEN atiende anualmente un gran número de casos de leucemias en niños, adolescentes y adultos, que son potenciales candidatos a trasplante de médula ósea; además, del grupo de pacientes que padecen de linfoma, mieloma múltiple y tumor de células germinales, razón por la cual desde abril de 2012 se viene trabajando el programa institucional de fortalecimiento de trasplante de médula ósea y células hematopoyéticas y en julio de 2012 se concluyó la implementación del Servicio de Protección al Inmuno Neutropénico (SEPIN) y trasplante de médula ósea (TAMO) del INEN, acreditada oficialmente por el MINSA, donde inicialmente se están atendiendo a pacientes adolescentes y adultos, para posteriormente atender a niños. Este servicio especializado está a disposición de la población peruana en general y a la fecha se han realizado cuatro trasplantes autólogos de células progenitoras hematopoyéticas que han sido financiados por el estado en el marco del Plan Esperanza dentro del convenio INEN y FISSAL al ser pacientes en condiciones de pobreza y extrema pobreza.

Desde junio del año 2012 se han incluido en este programa cien pacientes potenciales candidatos a trasplante de médula ósea (53 niños y adolescentes y 47 adultos); se han realizado 391 pruebas de histocompatibilidad mediante la tipificación de 5 locus a 32 pacientes (18 pediátricos y 14 adultos) con sus probables donantes emparentados. Se ha sistematizado los procedimientos para la atención de los pacientes que requieren los servicios de laboratorio de histocompatibilidad y criopreservación en coordinación con el SIS. Asimismo, el programa cuenta con un componente nacional, dentro del modelo de intercambio prestacional con EsSalud, para los trasplantes de médula ósea en población pediátrica, que por el momento no se realizan en el INEN, y un componente internacional de transferencia de pacientes para trasplantes de médula ósea no emparentados fuera del país. Por el intercambio prestacional con EsSalud ya se ha realizado un trasplante de médula ósea emparentado en un paciente pediátrico del INEN y se siguen coordinando para otros pacientes pediátricos que califiquen para este tipo de trasplante y cuenten con donantes emparentados compatibles, buscando continuidad en la atención de los pacientes. El desarrollo del sistema de soporte integral en el programa institucional de fortalecimiento de trasplante de médula ósea y células hematopoyéticas ha permitido que dentro del componente internacional se establezca el convenio entre el SIS-FISSAL y el Jackson Memorial Hospital de la Universidad de Miami, con el cual el INEN se encuentra trabajando intensamente la sistematización de la trasferencia de pacientes con donante compatible no emparentado detectado mediante el registro electrónico de la red internacional de banco de médula ósea de la mencionada universidad.

\section{CONCLUSIONES}

El cáncer es un problema de salud pública en Perú. En el INEN se han desarrollado e implementado estrategias 
para la atención de este problema, es así que se han tomado medidas de carácter nacional e institucional. A nivel nacional destaca la participación del INEN evidenciado en la descentralización de los servicios de atención oncológica especializados, con la creación de los IREN Norte e IREN Sur (en Trujillo y Arequipa), próximamente en Huancayo e lquitos, así como las unidades oncológicas y los preventorios de cáncer en Lima y otras regiones del país. A nivel institucional se debe mencionar la implementación del programa institucional de fortalecimiento de trasplante de médula ósea y células hematopoyéticas (TAMO) y el servicio de protección al inmuno neutropénico (SEPIN) acreditada oficialmente por el MINSA, el cual atendía inicialmente solo adolescentes y adultos, actualmente atiende también a población pediátrica.

Por otra parte, dentro de una política de estado integradora y articulada con el sector salud, y gracias al financiamiento del FISSAL, se pudo integrar los ejes estratégicos básicos para una atención integral del cáncer en la población de menores recursos, con lo cual vio la luz el Plan Nacional para la Atención Integral del Cáncer y el Mejoramiento del Acceso a los Servicios Oncológicos en el Perú, denominado "Plan Esperanza". El cual pone énfasis en la prevención de las enfermedades oncológicas más frecuentes, en la promoción de la salud y en la protección financiera de la población de menores recursos. Es necesario mencionar que uno de los pilares fundamentales en el cual se sostendría el Plan Esperanza, es el Programa Estratégico Presupuestal de Prevención y Control del Cáncer que ha sido diseñado, desarrollado e implementado por el INEN desde el año 2011, con una ejecución presupuestal y cumplimiento de las metas en forma sobresaliente. La conducción de este tipo de programas por un ente técnico especializado como es el INEN, permite mejores oportunidades para un trabajo operativo más dinámico y efectivo con un enfoque basado en experiencias especializadas y conocimientos profundos sobre la realidad de la enfermedad. Por todo lo expuesto, no se puede negar que el INEN cumple un rol importante en el manejo del cáncer como problema de salud pública en el Perú, procurando mantener en todo momento un alto nivel científico tecnológico, pero además brindando una atención oportuna y de calidad, integrando los conceptos de equidad y respeto a la dignidad del ser humano.

Contribuciones de autoría: TV y MS han participado en la concepción y diseño del artículo; TV, MS y JA participaron en la redacción; todos los autores participaron de la obtención de datos, revisión crítica y aprobación de la versión final.

Fuentes de financiamiento: autofinanciado.

Conflictos de interés: TV es jefa institucional del Instituto Nacional de Enfermedades Neoplásicas, todos los demás autores laboran en dicha institución.

\section{REFERENCIAS BIBLIOGRÁFICAS}

1. Perú, Instituto Nacional de Enfermedades Neoplásicas (INEN). Resumen de Indicadores de Salud 2012 [Internet]. Lima: INEN; 2012 [citado el 12 de enero del 2013]. Disponible en: http://www.inen.sld.pe/portal/ documentos/pdf/estadistica/datos_ estadisticos/06032013 RESUMEN INDICADORES_INTRANET.pdf

2. Perú, Instituto Nacional de Estadística e Informática (INEI). Censos Nacionales 2007: XI de Población y VI de Vivienda. Lima: INEI; 2008.

3. Perú, Ministerio de Salud, Dirección General de Epidemiología. Indicadores de Mortalidad 2011 [Internet]. Lima: Minsa; c2013 [citado el 12 de enero del 2013]. Disponible en: http:// www.dge.gob.pe/Asis/indbas/2011/ mortalidad.pdf
4. Perú, Instituto Nacional de Estadística e Informática (INEI). Condiciones de vida en el Perú [Internet]. Lima: INEI; c2013 [citado el 12 de enero del 2013]. Disponible en: http://www.inei.gob. pe/web/Biblioinei/BoletinFlotante. asp?file $=14486 . p d f$

5. Baider L. Cáncer y familia: aspectos teóricos y terapéuticos. Int $\mathrm{J}$ Clin Hlth Psyc. 2003;3(1):505-20.

6. Perú, Congreso de la República. Ley $\mathrm{N}^{\circ}$ 28343: Ley que declara de interés y necesidad pública la descentralización de los servicios médicos oncológicos. 09 de setiembre del 2004.

7. República del Perú. Decreto Supremo $\mathrm{N}^{\circ}$ 001-2007-SA. Reglamento de Organización y Funciones del Instituto nacional de Enfermedades Neoplásicas - INEN. Título I. De las Disposiciones Generales. 10 de enero del 2007.
8. Perú, Ministerio de Salud. Resolución Ministerial $N^{\circ}$ 030-2007/MINSA. Plan Nacional para el Fortalecimiento de la Prevención y Control del Cáncer. 11 de enero de 2007.

9. Perú, Ministerio de Salud. Resolución Ministerial N ${ }^{\circ}$ 589-2007 /MINSA. Plan Nacional Concertado de Salud. 20 de julio del 2007.

10. República del Perú. Decreto Supremo $\mathrm{N}^{\circ}$ 016-2009-SA. Plan Esencial de Aseguramiento en Salud - PEAS. 28 de noviembre del 2009.

11. Perú, Congreso de la República. Ley $N^{\circ}$ 29626: Ley de Presupuesto del Sector Público para el Año Fiscal 2011. 09 de diciembre del 2010.

12. Luxardo N. Entre la narrativa y la vivencia: lecturas a partir del cáncer. Estudios Sociales. 2008;(4):95-114. 
13. Luxardo N. Tejiendo tramas para explicar el cáncer (Argentina). Mitológicas. 2006;21:55-67.

14. Ritzer G. Teoría Sociológica contemporánea. México: McGraw Hill; 1993.

15. Blumer H. Las Actitudes y el acto social. En el interaccionismo simbólico. Barcelona: 1982.
16. República del Perú. Decreto Supremo $\mathrm{N}^{\circ}$ 009-2012-SA. Declaran de interés nacional la Atención Integral del Cáncer y Mejoramiento del acceso a los Servicios Oncológicos en el Perú y dictan otras medidas. 03 de noviembre del 2012.

17. Frosch DL, Kaplan RM. Shared decision making in clinical medicine: past research and future directions. Am J Prev Med. 1999; 17(4):285-94.

Correspondencia: Tatiana Vidaurre Rojas Dirección: Av. Angamos 2520. Lima 34, Perú. Teléfono: (511) 2016500

Correoelectrónico: tvidaurre@inen.sld.pe

\section{Suscríbase en forma electrónica y gratuita a los contenidos de la Revista Peruana de Medicina Experimental y Salud Pública, ingrese a WWW.ins.gob.pe, seleccione el ícono de la revista y envíenos sus datos.}

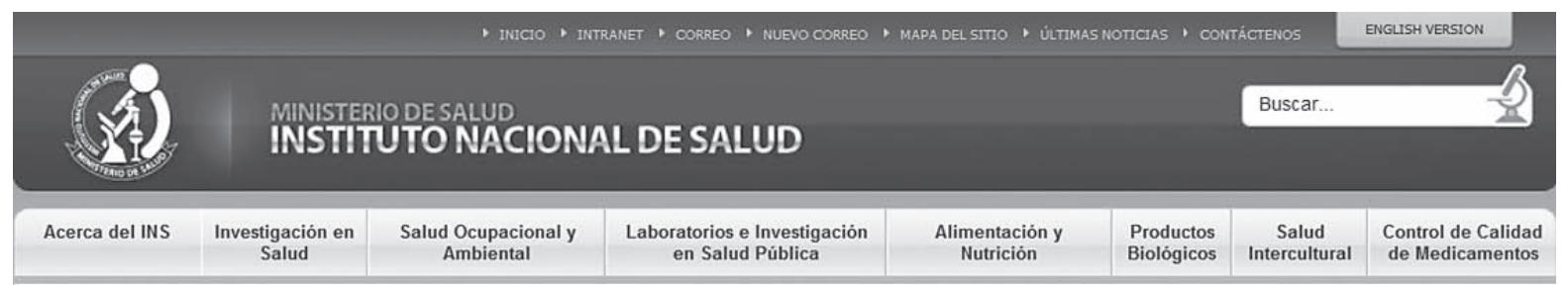

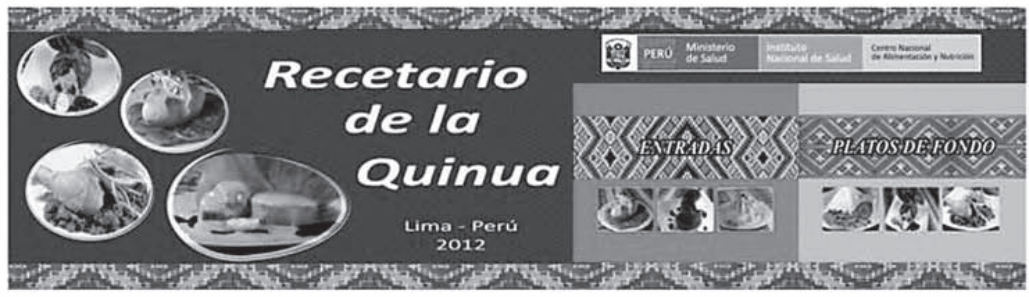

ÚLTIMAS INVESTIGACIONES Y EVIDENCIAS CIENTíFICAS Ver Todas

Procedimientos para la obtención, conservación y transporte de muestras para el diagnóstico de tos ferina

Fuente: Laboratorio de IRAS, Instituto Nacional de Salud

Ver detalle

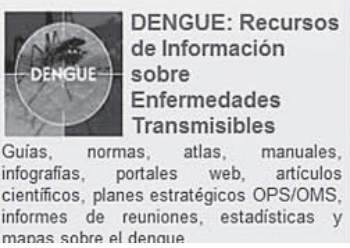

GALERÍA

VerTodas

Imagen del dia 2013

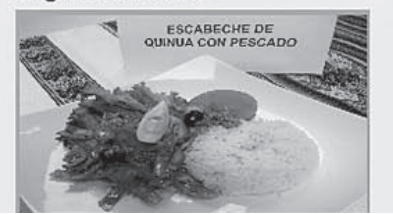

Q Portal del Transparencia

Gestión de calidad

6 Directorio Institucional

1.26 marzo del 2013

\. Agregar a favoritos

2. Enviar a un amigo

Enlaces de Interes

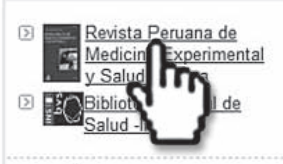

Ver más 